\title{
Stammzelltherapie schwerer Autoimmunkrankheiten
}

\author{
M. Hensel \\ C. Fiehn \\ A. D. Ho
}

Stem Cell Therapy for Severe Autoimmune Diseases

\section{Zusammenfassung}

Trotz Einsatz aller konventioneller (immunsuppressiver) Therapieformen kommt es in manchen Fällen schwer verlaufender Autoimmunerkrankungen zum Fortschreiten der Erkrankung, irreversiblen Organschäden und Todesfällen. Zusätzlich kann langdauernde immunsuppressive Therapie, v.a. mit Cyclophosphamid und Kortikosteroiden, zu schweren Nebenwirkungen einschließlich einer hohen Rate an Sekundärneoplasien führen. Daher wird seit mehreren Jahren im Rahmen von experimentellen Studienprotokollen der Nutzen einer kurzfristigen dosiseskalierten Immunsuppression in Form der Hochdosischemotherapie (HDCT) mit autologer Stammzelltransplantation bei ansonsten therapierefraktären Patienten erprobt. Im Mittelpunkt dieser Bemühungen stehen bisher Patienten mit progressiver systemischer Sklerose, systemischem Lupus erythematodes, Rheumatoidarthritis, multipler Sklerose, Autoimmunhämolyse und Autoimmunthrombopenie. Auch Patienten mit primär systemischen Vaskulitiden (PSV) könnten geeignete Kandidaten für die HDCT sein. Es müssen die Patienten ausgewählt werden, bei denen die Schwere der Erkrankung, bzw. die krankheitsassoziierte Mortalität die Toxizität der HDCT erheblich überwiegt. Grundsätzlich sollten für die ASCT Patienten ausgewählt werden, deren Erkrankung therapierefraktär, aktiv und progredient ist, und bei denen ein hohes Risiko für bleibende Funktionseinschränkungen und hohe Mortalität trotz konventioneller Therapie besteht. Durch verbesserte Scoring-Systeme, die eine frühere Identifikation dieser Hochrisiko-Patienten schon in frühen Krankheitsstadien erlauben, kann zukünftig der Erfolg der Methode verbessert und die Risiken minimiert werden. Erste Publikationen sowie ei-

\section{Abstract}

Many patients with various autoimmune diseases fail to respond to conventional immunosuppressive therapy and develop irreversible organ damage. In some cases the complications might be fatal. Furthermore, prolonged immunosuppression with cyclophosphamide or corticosteroids often leads to long-term side effects, cumulative organ damage and development of secondary malignancies. Thus, short-term, high-dose immunosuppressive therapy with autologous stem cell transplantation (ASCT) might be an alternative for otherwise refractory patients. This concept has been used mainly for patients with scleroderma, systemic lupus, rheumatoid arthritis and multiple sclerosis, autoimmune hemolysis and thrombocytopenia. Patients with primary systemic vasculitis (PSV) also seem to be suitable candidates for high dose chemotherapy (HDCT). The patients must be identified in whom disease severity and disease-related mortality overweighs the toxicity of the high-dose chemotherapy. Only patients with active, refractory and progressing disease who are at high risk for cumulative toxicity due to conventional therapy should be eligible for ASCT. Careful patient selection with the help of scoring systems and determination of the optimal time in the course of disease are now the major goals in the approach to HDCT. First reports together with our own single center experience in HDCT with a limited number of patients with severe PSV showed that long-term remissions even in patients refractory to conventional immunosuppression are possible with acceptable toxicity. For the next years, controlled trials should be considered to determine the potential and risks of ASCT compared to conventional immunosuppressive therapy. 
gene Erfahrungen bei wenigen, ausgewählten Patienten mit schwer verlaufenden PSV und anderen Autoimmunerkrankungen zeigen, dass langfristige Remissionen auch in vormals therapierefraktären Fällen möglich sind bei akzeptabler Toxizität. Zukünftig können allein durch multizentrische Phase-III-Studien der Nutzen und die Risiken der Stammzelltransplantation im Vergleich zur konventionellen Therapie geklärt werden.

\section{Einleitung}

Bei vielen Autoimmunerkrankungen kann die Krankheitsaktivität ausreichend durch eine konventionelle immunsuppressive oder immunmodulatorische Therapie beherrscht werden. Trotzdem werden immer wieder schwer verlaufende Fälle beobachtet, bei denen die konventionelle Therapie versagt, die Krankheit fortschreitet und zu irreversiblen Organschäden mit massiv eingeschränkter Lebensqualität und teilweise auch zum Tod führt. So sind z.B. schwere Verlaufsformen der systemischen Sklerose mit einer 5-Jahres-Mortalität von 45-50\% behaftet, ohne dass hier effektive Behandlungsmaßnahmen zur Verfügung stehen, die den Krankheitsfortschritt oder die Fibrosierung aufhalten können. Lediglich durch Cyclophosphamid konnte in einigen, nicht randomisierten Studien gezeigt werden, dass bestimmte klinische Parameter zumindest vorübergehend gebessert werden konnten [1-3].

Obwohl die Langzeitprognose von Patienten mit systemischem Lupus erythematodes (SLE) inzwischen durch frühere Diagnosen und den konsequenten Einsatz von Glukokortikoiden und Immunsuppressiva wie Cyclophosphamid im Vergleich zu früher sehr günstig geworden ist (5-Jahres-Überleben $>90 \%$ ), gibt es weiterhin eine kleine Gruppe von Lupus-Patienten, bei denen die Erkrankung einen sehr ungünstigen Verlauf nimmt mit sehr schlechtem Ansprechen auf die Standardtherapie [4-6]. Ähnliches trifft auch für eine andere, sehr weit verbreitete, den Autoimmunerkrankungen zugeordnete Krankheit, die multiple Sklerose (MS) zu. Hier ist die Lebenserwartung um ca. 10 Jahre vermindert, verglichen mit der gesunden Normalbevölkerung gleichen Alters [7]. Gewisse Erfolge in der Behandlung konnten durch den Einsatz von Interferon- $\beta$ und Glatiramer-Acetat (Copaxone) erreicht werden $[8,9]$. Es handelt sich allerdings bei der MS um eine sehr heterogene Krankheitsgruppe mit möglicherweise unterschiedlicher Ätiologie. Vor allem in der sekundär chronisch-progressiven Phase der Erkrankung sind die genannten Medikamente nur noch sehr eingeschränkt wirksam. Lediglich durch Mitoxantron konnte hier zuletzt in Studien ein günstiger Einfluss auf die Progression des Grades der Behinderung und dem Auftreten von ZNS-Herden nachgewiesen werden $[10,11]$. Bei einem Teil der Patienten kann auch durch diese Behandlung das Fortschreiten der Erkrankung nicht aufgehalten werden.

Für all diese Patienten mit therapierefraktären Autoimmunerkrankungen stellt die immunoablative Chemotherapie mit nachfolgender Stammzelltransplantation eine neue therapeutische Option dar. Sie basiert auf einer maximalen Eskalation der immunosuppressiven zytotoxischen Substanzen („Konditionie- rung“) mittels Chemotherapie und/oder Ganzkörperbestrahlung (TBI). Diese Behandlung ist nicht nur immunoablativ, sondern auch myeloablativ, d.h. die der zytotoxischen Therapie ausgesetzten Stammzellen des Knochenmarks werden unwiederbringlich zerstört. Daher ist die Infusion fremder hämatopoetischer Stammzellen (im Falle der allogenen Stammzelltransplantation) oder Rückinfusion von zuvor gewonnenen und tiefgefrorenen autologen Stammzellen erforderlich. Diese Methode wird schon seit über 30 Jahren in Form der Knochenmarktransplantation bei verschiedensten hämatologischen und onkologischen malignen Erkrankungen eingesetzt. Seitdem aber in den letzten 15 Jahren immer häufiger peripheres Blut als Quelle der Stammzellgewinnung verwendet wird (aus dieser Methode resultiert eine kürzere Aplasiedauer und eine geringere Komplikationsrate), wird die Behandlung inzwischen „hämatopoetische Stammzelltransplantation“ genannt.

Grundlage des Einsatzes der Stammzelltransplantation bei Autoimmunerkrankungen ist die in den vergangenen Jahrzehnten gelegentlich gemachte Beobachtung, dass Patienten mit Autoimmun- oder entzündlich rheumatischen Erkrankungen, die an einer hämatologischen Neoplasie litten und deswegen mit einer Stammzelltransplantation behandelt wurden, eine Remission ihrer Autoimmunerkrankung erfuhren [12]. Darüber hinaus konnten in einer Reihe von tierexperimentellen Arbeiten die erfolgreiche Behandlung von Autoimmunerkrankungen mittels allogener oder autologer Stammzelltransplantation nachgewiesen werden [13-17].

Im Folgenden beschreiben wir die Methodik der Stammzellgewinnung, der Hochdosistherapie und der Stammzelltransplantation, die hypothetischen Wirkmechanismen sowie die bisherigen klinischen Erfahrungen bei verschiedenen Krankheitsbildern.

\section{Stammzellquelle und Mobilisierungsregime}

Prinzipiell gibt es zwei Quellen der Stammzellgewinnung: Knochenmarkentnahme in Narkose oder Sammlung peripherer Blutstammzellen mittels Leukapherese. Bei hämatologisch-onkologischen Neoplasien hat sich die Verwendung peripherer Blutstammzellen durchgesetzt. Aus ihnen entwickeln sich neben Erythrozyten und Thrombozyten auch die Zellen des Immunsystems. Sie können nach einer geeigneten Mobilisierungsbehandlung mit Kolonie-stimulierenden Faktoren (insbesondere G-CSF) alleine oder in Kombination mit Chemotherapie (Stammzellsammlung im Rebound bei der Erholung des Knochenmarks) aus dem peripheren Blut mittels Leukapherese gewonnen werden. Aus dem peripheren Blut gewonnene Stammzellen führen zu einer schnellen und kompletten hämatopoetischen Rekonstitution nach Hochdosistherapie. Dieses Verfahren wird heutzutage vor allem bei Patienten mit Autoimmunerkrankungen kombiniert mit einer Reinigung des gesammelten Stammzelltransplantates von ausgereiften T-Zellen, um so das Rückfallrisiko zu verringern. Bisher existieren jedoch keine Daten, die eine Überlegenheit der Transplantation T-Zell-depletierter Stammzelltransplantate gegenüber unselektierten Transplantaten zeigen. 
Im Rahmen des Heidelberger Studienprotokolls wird nach einer initialen Induktions- bzw. Mobilisierungschemotherapie mit Cyclophosphamid $\left(2 \mathrm{~g} / \mathrm{m}^{2}\right)$ die Stammzellsammlung in der Phase des Wiederanstiegs der Leukozyten durchgeführt. Sollte es z.B. aufgrund zu starker Vorbehandlungen nicht möglich sein, ausreichend periphere Blutstammzellen zu gewinnen, folgt ein weiterer Zyklus Cyclophosphamid in einer Dosis von $4 \mathrm{~g} / \mathrm{m}^{2}$ und erneuter Stammzellsammlung [18]. Zur Mobilisierung der Stammzellen erhalten die Patienten im Anschluss an die Cyclophosphamid-Infusionen täglich Injektionen mit dem Granulozyten-Wachstumsfaktor G-CSF. Aus dem Leukaphäreseprodukt werden ex vivo mittels einer speziellen Technik die T-Zellen weitgehend eliminiert (Anreicherung von CD34-positiven Stammzellen, Baxter Isolex 300 SA Magnetic Cell Separation System). Innerhalb von 4-6 Wochen nach erfolgreicher Stammzellsammlung folgt die eigentliche Hochdosis-Chemotherapie mit Melphalan $\left(200 \mathrm{mg} / \mathrm{m}^{2}\right)$. Anschließend werden die zuvor kryokonservierten Stammzellen den Patienten zurückgegeben. Die Rückgabe erfolgt als i.v. Injektion. Die Stammzellen migrieren selbstständig ins Knochenmark, um dort die Blutbildung zu übernehmen.

Cyclophosphamid ist das effektivste Immunsuppressivum zur primären Remissionsinduktion bei primär systemischen Vaskulitiden und ist auch ein wesentlicher Bestandteil der Therapie vieler anderer Autoimmunerkrankungen mit schweren Organbeteiligungen wie dem SLE, der progressiven systemischen Sklerose u. a. Darüber hinaus ist die Mobilisierungstherapie mit Cyclophosphamid sicher durchführbar und führt bei Patienten mit Autoimmunerkrankungen zur erfolgreichen Stammzellsammlung [19]. Die Verwendung von Cyclophosphamid in der Mobilisierungstherapie der Stammzellsammlung hat daher auch einen erheblichen Effekt auf das therapeutische Ziel der Langzeitremissionsinduktion.

\section{Konditionierungsregime}

Das Konditionierungsregime dient der langfristigen Elimination autoreaktiver T-Zellen. Es werden verschiedene, meist aus der Hämato-Onkologie entlehnte Konditionierungsprotokolle verwendet. Gebräuchlichste Substanzen oder Methoden sind Hochdosis-Cyclophosphamid + Antilymphozytenglobulin + Ganzkörperbestrahlung (TBI). Verwendung finden auch Konditionierungsschemata, wie sie in der Leukämie- und Lymphomtherapie erprobt sind (Busulphan/Cyclophosphamid; „BEAM“; Melphalan). Es ist jedoch bisher unklar, welches dieser myeloablativen Regime am ehesten geeignet ist, das Immunsystem einschließlich der Memoryzellen am effektivsten zu eradizieren.

Zur Konditionierung verwenden wir in Heidelberg das Melphalan-Regime (Abb.1). Es ist myeloablativ, ohne dass eine zusätzliche Ganzkörperbestrahlung, die die Toxizität erhöhen würde, notwendig wäre. Es wird seit Jahrzehnten erfolgreich bei hämatologischen Erkrankungen im Rahmen der autologen und allogenen Transplantation eingesetzt. Vor allem aber ist die Toxizität geringer als bei den meisten anderen Konditionierungsschemata und es existieren Erfahrungen bei Patienten mit terminaler Niereninsuffizienz i.R. der Therapie der AL-Amyloidose. Die Toxizität wurde im Rahmen von Hochdosis-Programmen bei Patienten mit multiplem Myelom evaluiert [20]. Es konnte gezeigt werden, dass die Rate an Sekundärneoplasien (z.B. myelodysplastisches Syndrom, akute Leukämie) geringer war als bei lang andauernder Standard-Chemotherapie [21].

\section{Hypothesen zum Wirkmechanismus}

Durch die myeloablative bzw. immunoablative Hochdosis-Chemotherapie sollen aberrante, auto-aggressive immunkompetente Zellen langfristig beseitigt werden. Allerdings zeigen die bisher gewonnenen Daten an über 400 Patienten, die im Rahmen des Hochdosis-Protokolls behandelt wurden, dass immunoablative Verfahren wahrscheinlich nicht in der Lage sind, auch die letzte auto-reaktive bzw. Memory-T-Zelle zu vernichten. Wahrscheinlich ist dies auch gar nicht nötig. Vielmehr erscheint es durch dieses Verfahren möglich zu sein, dass durch „Debulking“ des Pools der autoreaktiven Zellen das Ungleichgewicht zwischen krankheitsauslösender „aberranter“ Immunreaktion und krankheitsunterdrückenden Toleranzmechanismen durch die Hochdosistherapie wiederhergestellt werden kann [22,23]. Die klinische Autoimmunität wird inzwischen von einigen Autoren eher verstanden als Ungleichgewicht zwischen „krankheitsinduzierenden, aberranten“ Immunantworten, provoziert durch exogene Antigene und „krankheitsunterdrückenden“ peripheren Toleranzmechanismen. Durch die myeloablative bzw. immunoablative Hochdosistherapie werden die autoreaktiven Zellen zwar nicht ganz eliminiert, jedoch vorübergehend sehr stark reduziert. Im Rahmen der Rekonstitution des Immunsystems in den Wochen und Monaten nach Stammzelltransplantation soll dann diese Balance wiederhergestellt werden. Zumindest wird ein solcher Mechanismus diskutiert bei der Anti-T-Zell-Antikörpertherapie [16,24]. Einige Autoren vermuten, dass weniger die Induktion von Selbsttoleranz durch Rekapitulation der lymphozytären Ontogenese entscheidend für die Wirkung der autologen Stammzelltransplantation ist, als vielmehr die lang anhaltende Suppression von autoreaktiven Lymphozyten. Dafür spricht auch, dass im humanen autologen Setting die ontogenetische Rekapitulation bisher nur für B-Lymphozyten, nicht jedoch für T-Lymphozyten gezeigt werden konnte $[25,26]$. Bei der allogenen Stammzelltransplantation wird das gesamte Immunsystem ersetzt durch das Immunsystem des Spenders und hierdurch werden zumindest hypothetisch alle autoreaktiven Lymphozyten beseitigt. Von manchen Autoren wird diese Situation gelegentlich als „Graft-versus-Autoimmunity-Effekt“ beschrieben [27]. Jedoch zeigen unsere eigenen klinischen Erfahrungen an kleinen Patientenzahlen sowie einzelne Fallberichte, dass auch nach allogener Stammzell- bzw. Knochenmarktransplantation Rezidive z.B. der Rheumatoidarthritis beobachtet werden $[28,29]$.

\section{Bisherige klinische Erfahrungen}

Die anlässlich eines internationalen Symposiums (European Group for Blood and Marrow Transplantation Working Party for Autoimmune Diseases) in Basel im Oktober 2000 zusammen getragenen Daten über alle bisher transplantierten und registrierten Patienten zeigen eine hohe Ansprechrate bei verschiedenen Autoimmunerkrankungen [30]. In dieser von der European Group for Blood and Marrow Transplantation (EBMT) und der 
European League Against Rheumatism (EULAR) zusammen mit nordamerikanischen Arbeitsgruppen zusammengestellten Datenbank sind inzwischen mehr als 400 transplantierte Patienten zusammengefasst. Im Mittelpunkt der Bemühungen standen bisher Patienten mit systemischer Sklerose, systemischem Lupus erythematodes, rheumatoider Arthritis und multipler Sklerose.

\section{Systemische Sklerose}

Die zweithäufigste Indikation für eine autologe Stammzelltransplantation im Rahmen der EBMT/EULAR-Datenbank ist die systemische Sklerose (SSc). Die Mortalität der Patienten mit SSc ist gegenüber der Normalbevölkerung erhöht [31,32]. Altman berichtete 1991 über eine Überlebensrate von 80\% nach 2 Jahren, 50\% nach 8,5 Jahren und 30\% nach 12 Jahren. In einer großen schwedischen Kohorte mit 249 Patienten mit systemischer Sklerose betrug die 5-Jahres- und 10-Jahres-Überlebensrate der gesamten Gruppe 86\% bzw 69\% [33]. Die diffuse Form der Sklerose hat die schlechteste Prognose mit einer 10-Jahres-Überlebensrate von ca. $55 \%$ verglichen mit $70 \%$ bei der limitierten Form [34]. Bei der SSc sind viele Fragen hinsichtlich Ätiologie und Pathogenese, insbesondere hinsichtlich Beteiligung des Immunsystems, noch ungelöst. Die Beobachtung, das immunsuppressive Therapie in den meisten Fällen die Fibrosierung des Gewebes nicht rückgängig machen oder aufhalten kann, spricht dafür, dass dieser Prozess zwar durch Autoimmunphänomene angestoßen wird, jedoch bald einen vom Immunsystem unabhängigen Verlauf nimmt und automatisch fortschreitet. Angesichts der oben beschriebenen sehr schlechten Prognose der SSc und dem Fehlen effektiver Behandlungsmethoden wurde hier frühzeitig der Einsatz der Hochdosistherapie mit Stammzelltransplantation evaluiert [35]. Bei der SSc ist es besser als bei anderen Autoimmunerkrankungen möglich, Patienten mit sehr schlechter Prognose mit stark verkürzter Lebenserwartung anhand von etablierten Prognosekriterien zu identifizieren.

Es existieren inzwischen mehrere Publikationen zur Effektivität und Mortalität der Stammzelltransplantation bei Patienten mit SSc. Die Analyse der ersten 41 Patienten der EBMT/EULAR-Datenbank mit einem medianen Follow-up von 12 Monaten ergab eine signifikante Besserung des Skin-Scores und zumindest einen Trend zur Stabilisierung der Lungenfunktion [36]. Beunruhigend war eine mit $17 \%$ sehr hohe transplantationsassoziierte Mortalität (TRM). Es handelte sich bei dieser Kohorte allerdings um die ersten, mit dieser Diagnose transplantierten Patienten im Rahmen der EBMT/EULAR-Datenbank. Die Analyse der später registrierten und transplantierten Patienten ergab einen Abfall der TRM auf 12,5\% [30]. Werden nur die Patienten analysiert, die die aktuellen EBMT/EULAR-Einschlusskriterien für Transplantationsstudien erfüllen, so liegt die TRM nur noch bei 7,7\%. Die größte bisherige Phase-II-Studie wurde in verschiedenen nordamerikanischen Zentren durchgeführt und schloss $19 \mathrm{~Pa}-$ tienten mit SSc und ungünstiger Prognose ein [37]. In der frühen Studienphase verstarben 2 Patienten an Lungenversagen. Ein Patient verstarb an einem EBV-assoziierten Lymphom, ein weiterer an Krankheitsprogress. Bei den übrigen Patienten besserte sich in den meisten Fällen sowohl der Rodnan-Skin-Score als auch weitere krankheitsassoziierte Parameter (health assessment questionnaire disability index, mHAQ-DI) signifikant. Bei einem medianen Follow-up von 14,7 Monaten betrug die geschätzte 2-Jahres-Überlebenrate 79\%. Die Funktionen lebenswichtiger Organe wie Lunge, Niere und Herz blieben meist stabil, jedoch kam es in einigen Fällen zu einer Reaktivierung der Krankheitsaktivität im Verlauf. Diese Daten zeigen erstmals, dass sowohl die Hautsklerosierung wieder rückgängig gemacht werden kann, als auch eine Stabilisierung der Organfunktionen und Besserung körperlicher Funktionen bei ansonsten stark behinderten Patienten mit SSc möglich ist.

Die hohe TRM wird zumindest zum Teil auf die Patientenselektion zurückgeführt, da Hochrisikopatienten präferenziell behandelt wurden. Hier scheinen pulmonale Hypertonie, fortgeschrittene Lungenfibrose und aktive Herzerkrankung die wichtigsten Risikofaktoren zu sein.

Eine erste kontrollierte, randomisierte Studie bei Patienten mit schwerer systemischer Sklerose wurde inzwischen gestartet (ASTIS-Trial, Autologous Stem Cell Transplantation International Scleroderma; Protokoll erhältlich über www.astistrial.com). Im Rahmen dieser Studie werden strikte Kriterien gefordert, die Patienten mit fortgeschrittener Beteiligung innerer Organe ausschließen und so auf eine günstigere TRM hoffen lassen. So werden z.B. Patienten mit einem mittleren pulmonalarteriellen Druck von $>50 \mathrm{~mm} \mathrm{Hg}$, Patienten mit einer Kreatininclearance von $<40 \mathrm{ml} / \mathrm{min}$ und Patienten mit einer Ejektionsfraktion des Herzens $<45 \%$ ausgeschlossen. Gleichzeitig ist aber neben einer diffusen kutanen Beteiligung der Sklerodermie eine Organbeteiligung des Herzens, der Lunge oder der Niere Voraussetzung für die Aufnahme in die Studie.

\section{Multiple Slklerose}

Die multiple Sklerose (MS) ist eine schwere demyelinisierende, immunvermittelte Erkrankung des zentralen Nervensystems (ZNS), die hauptsächlich junge Erwachsene befällt und in der Mehrzahl der Fälle zu physischer und psychischer Funktionseinschränkung führt [7]. 10 Jahre nach Beginn der Erkrankung entwickeln $50 \%$ der Patienten einen chronisch progressiven Verlauf. Manche Patienten sprechen überhaupt nicht an auf die Standardtherapien (Interferon beta, Glatiramer-Acetat). Immunsuppressive Behandlungsformen, die in der Regel als Second-lineTherapie bei MS-Patienten angewendet werden, weisen auch nur bei einem Teil der Patienten eine Wirksamkeit auf.

Die MS stellt die größte Krankheitsgruppe innerhalb der EBMT/ EULAR-Datenbank dar. Es sind inzwischen über 100 Patienten registriert [27]. Die meisten Zentren richten sich bei der Patientenselektion nach den Empfehlungen eines Konsensusmeetings 1998 in Mailand [38]. Meist wurden Patienten mit sekundär progressiver MS mit schweren Behinderungen (Kurtzke-EDSS zwischen 5,0 und 8,0) eingeschlossen. Alle bisherigen Publikationen berichten übereinstimmend über einen günstigen Effekt auf die weitere Krankheitsprogression. Die bisherige Auswertung der EBMT-Daten [30] von 85 Patienten und einem medianen EDSSScore von 6,5 bei einem medianen Follow-up von 16 Monaten ergab eine Krankheitsstabilisierung bei der Mehrzahl der Patienten. Bei $21 \%$ der Patienten konnte eine Verbesserung der neurologischen Funktion erreicht werden mit einem Rückgang des 
EDSS-Behinderungsscores $>1,0$ Punkte. Das progressionsfreie Überleben betrug nach 3 Jahren $68 \%$. Auch die MRI-Daten zeigten einen günstigen Einfluss der Stammzelltransplantation auf die Krankheitsaktivität bei der Mehrzahl der Patienten. Die TRM betrug $6 \%$. Auch bei dieser Indikation müssen die günstigen $\mathrm{Er}-$ gebnisse durch prospektive, randomisierte Studien validiert werden. Eine solche Studie wird in Kürze von der EBMT gestartet (ASTIMS Trial - Autologous Stem Cell Transplantation International Multiple Sclerosis Trial. www.ebmt.org).

\section{Systemischer Lupus erythematodes}

Die Mehrzahl der Patienten mit SLE hat inzwischen durch frühere Diagnosen und dem konsequenten Einsatz von Glukokortikoiden und Immunsuppressiva wie Cyclophosphamid im Vergleich zu früher eine günstigere Langzeitprognose mit einer 5-JahresÜberlebensrate von $90 \%$. Allerdings gibt es weiterhin eine kleine Gruppe von Lupus-Patienten, bei denen die Erkrankung einen sehr ungünstigen Verlauf nimmt mit sehr schlechtem Ansprechen auf die Standardtherapie. Die EBMT/EULAR-Datenbank beinhaltet bisher 23 transplantierte Patienten mit SLE [39]. Einige dieser Patienten sowie weitere außerhalb der Datenbank sind in kleinen Fallzusammenstellungen publiziert worden [40-43]. Die Einschlusskriterien variieren zwischen den einzelnen Zentren. Meist jedoch wurden Patienten eingeschlossen 1) mit bioptisch gesicherter WHO-Klasse-III- und -IV-Glomerulonephritis, die nicht auf Cyclophosphamid-Pulstherapie angesprochen haben, 2) die eine begeleitende Vaskulitis oder schwere ZNS-Beteiligung aufwiesen, die nicht durch Kortikosteroide oder Cyclophosphamid zu beherrschen war, 3) die therapierefraktäre schwere immunologisch vermittelte Zytopenien hatten und 4) die ein sog. katastrophales Antiphospholipid-Syndrom hatten. Die Arbeitsgruppe um Richard Burt in Chicago hat kürzlich die Ergebnisse der autologen Stammzelltransplantation bei inzwischen 15 Patienten mit Cyclophosphamid vorbehandeltem SLE und einer medianen Nachbeobachtungsphase von 36 Monaten [43] publiziert. Die Krankheitsaktivität verbesserte sich bei allen Patienten. Der SLEDAI-Score, der die Krankheitsaktivität des SLE misst, fiel auf 5 oder darunter bei 12 von 15 Patienten, was eine niedrige Krankheitsaktivität anzeigt. Komplement- und dsDNSSpiegel haben sich bei allen Patienten normalisiert. Auch die Organfunktionen hatten sich bei allen Patienten verbessert. Von den 12 Patienten, die länger als $1 \mathrm{Jahr}$ nachbeobachtet wurden, konnte bei 10 die immunsuppressive Therapie vollständig abgesetzt werden. Todesfälle der Stammzelltransplantation traten nicht auf, allerdings verstarb ein Patient an einer Komplikation der Mobilisierungstherapie.

\section{Rheumatoidarthritis}

Eine kürzlich publizierte retrospektive Analyse der Registry-Daten von 76 Patienten mit Rheumatoidarthritis aus 15 Zentren ergab ein initial günstiges Ansprechen der massiv vorbehandelten Patienten auf die autologe Stammzelltransplantation [44]. In $67 \%$ der Fälle besserte sich die Krankheitsaktivität um mindestens $50 \%$ gemäß den Kriterien des American College of Rheumatology. Allerdings musste bei den meisten Patienten die krankheitsmodifizierende Basistherapie (DMARDs) innerhalb von 6
Monaten nach Transplantation wieder angesetzt werden wegen eines Rezidivs. Es ist zu erwarten, dass angesichts dieser Daten und der guten Wirksamkeit neuer biologisch aktiver Substanzen wie den Anti-TNF-Wirkstoffen (Remicade ${ }^{\circledR}$, Enbrel ${ }^{\circledR}$ Humira ${ }^{\circledR}$ ) die Stammzelltransplantation zur Behandlung der RA keine große Rolle spielen wird.

\section{Primär systemische Vaskullitis}

Patienten mit primär systemischen Vaskulitiden (PSV) scheinen erfolgversprechende Kandidaten für die HDCT zu sein, da insbesondere in dieser Patientengruppe in vielen Fällen komplette, lang anhaltende Remissionen durch konventionelle immunsuppressive Behandlungen möglich sind. Gerade diese Möglichkeit der kompletten Remission durch eine intensivierte Chemotherapie (Induktions- oder Mobilisierungschemotherapie) ist seit Jahrzehnten eine essenzielle Voraussetzung für den Erfolg der HDCT und Stammzelltransplantation bei hämatologisch-onkologischen Erkrankungen. Daher ist zu vermuten, dass dies auch eine gute Basis für langfristige Remissionen durch HDCT bei PSV sein könnte.

Trotz der Therapie mit Kortikosteroiden und Cyclophosphamid, die seit einigen Jahrzehnten die Prognose der PSV dramatisch verbessert hat, gibt es auch im Jahr 2003 immer noch eine Subgruppe von Patienten mit PSV, die 1.) nicht geheilt werden können durch die Standardtherapie, 2.) eine langfristige Immunsuppression benötigen und 3.) die sogar entweder an der Progression der Erkrankung oder an der kumulativen Therapie-assoziierten Toxizität versterben. In den meisten größeren Studien konnte die 5-Jahres-Mortalität nicht unter $14-20 \%$ gesenkt werden $[45,46]$. Lediglich in einer Langzeit-Follow-up-Studie mit 155 Patienten mit WG wurde eine etwas günstigere Gesamtmortalität beobachtet [47]. Die Mortalitätsrate ist sowohl auf die Krankheitsaktivität als auch auf die Therapiefolgen zurückzuführen. Im Rahmen der europäischen Vaskulitisarbeitsgruppe EUVAS werden in mehreren Therapiestudien therapeutische Alternativen zur bisherigen Standardtherapie Cyclophosphamid zur Remissionsinduktion und -erhaltung und zum Vergleich der täglichen oralen mit der intravenösen Bolustherapie von Cyclophosphamid bei der Wegenerschen Granulomatose und mikroskopischen Polyangiitis untersucht [48]. Der erste Schritt der Behandlung primärer systemischer Vaskulitiden sollte in einer konventionellen Remissionsinduktion mit Cyclophosphamid und Glukokortikoiden bestehen. Nachdem eine Remission erreicht wurde, sollten mithilfe klinischer Scoring-Systeme die Patienten mit besonders schlechter Prognose identifiziert werden. Hier bieten sich mehrere Prognosescores an, vor allem FFS [49], BVAS [50] und DEI [51] welche in der Tab.1 näher dargestellt sind. Alle diese „Werkzeuge“ können dabei helfen, gerade die Patienten mit schwerer Erkrankung und schlechter Prognose zu identifizieren, die eine intensivere Immunsuppression einschließlich der ASCT benötigen. Nachdem eine Remission durch konventionelle Induktionschemotherapie erzielt wurde, kann bei Patienten mit einem FFS $>2$ (46\%ige Einjahresmortalität) oder einem initial hohen BVAS $(>20)$ der Einschluss in eine Hochdosisstudie erwogen werden, anstatt einer langfristigen Behandlung mit konventioneller Erhaltungstherapie. Basierend auf diesen Überlegungen wählen wir an unserer Institution derzeit 
Tab. 1 Vereinfachte Übersicht über die klinischen Scoring-Systeme für systemische Vaskulitis. Anzahl der möglichen Manifestationen in verschiedenen Organsystemen, welche in die Kalkulation des Scores einbezogen sind

\begin{tabular}{lllll}
\hline Organsysteme & $\begin{array}{l}\text { Aktivität } \\
\text { (FFS) }\end{array}$ & $\begin{array}{l}\text { Aktivität } \\
\text { (BVAS) }\end{array}$ & $\begin{array}{l}\text { Ausdehnung } \\
\text { (DEI) }\end{array}$ & $\begin{array}{l}\text { Schädigung } \\
\text { (VDI) }\end{array}$ \\
\hline HNO & - & $0-6$ & $0-1$ & $0-6$ \\
\hline Lunge & - & $0-5$ & $0-1$ & $0-7$ \\
\hline Niere & $0-2$ & $0-7$ & $0-1$ & $0-3$ \\
\hline Haut & - & $0-6$ & $0-1$ & $0-2$ \\
\hline Mukosa & - & $0-2$ & - & $0-1$ \\
\hline Auge & - & $0-5$ & $0-1$ & $0-7$ \\
\hline Gelenke/Muskel & - & $0-2$ & $0-1$ & $0-5$ \\
\hline ZNS & $0-1$ & $0-4$ & $0-1$ & $0-6$ \\
\hline $\begin{array}{l}\text { PNS } \\
\text { kardiovaskuläres }\end{array}$ & $0-1$ & $0-7$ & $0-1$ & $0-7 / 9$ \\
System & $0-1$ & $0-2$ & $0-1$ & $0-4$ \\
\hline $\begin{array}{l}\text { gastrointestinal } \\
\text { systemische }\end{array}$ & - & $0-5$ & $0-1$ & - \\
Manifestationen & & & $0-1$ & $0-1$ \\
\hline $\begin{array}{l}\text { Nebenwirkungen } \\
\text { der Therapie }\end{array}$ & - & - & - & $0-5$ \\
\hline \begin{tabular}{l} 
maximaler Score \\
\hline
\end{tabular} & $\mathbf{5}$ & $\mathbf{6 3}$ & $\mathbf{2 1}$ & $\mathbf{6 4}$ \\
\hline
\end{tabular}

FFS = Five factor score [49], BVAS = Birmingham vasculitis activity score [50], $\mathrm{VDI}=$ Vasculitis activity index [52], DEI = Disease extension index [51]

Patienten für eine Hochdosistherapie nach folgenden Kriterien aus:

- Patienten mit einer hohen Krankheitsaktivität und Beteiligung innerer Organe, gemessen an einem erhöhten FFS und BVAS

sowie eines oder mehrere der folgenden Kriterien:

- Patienten, die nicht auf Standard-Immunsuppression ansprechen,

- Patienten, die wiederholte Rückfälle mit zunehmenden Organschäden entwickeln,

- Patienten mit hohem Risiko für Organschäden als Folge langfristiger immunsuppressiver Therapie.

Die Erfahrung mit Hochdosistherapie bei onkologischen Patienten oder bei Patienten mit anderen Autoimmunerkrankungen wie multipler Sklerose oder Sklerodermie haben gezeigt, dass vor allem die frühe Identifikation der Risikopatienten, d.h. vor Auftreten schwerer Organschäden, entscheidend für den Erfolg dieses Verfahrens ist. Die Schädigung der Organe durch die Vaskulitis kann mithilfe des Vasculitis Damage Index (VDI) [52] der ebenso in der Tab. 1 dargestellt ist, gemessen werden. Hieraus resultieren folgende Ausschlusskriterien:

- Vasculitis Damage Index $>5$,

- links ventrikuläre Ejektionsfraktion < $45 \%$ des Normalwertes,

- unkontrollierte Herz-Rhythmusstörungen,

- pulmonale Diffusionskapazität $<40 \%$ des Solls,

- Ateminsuffizienz mit $\mathrm{PaO}_{2}<8 \mathrm{kPa}(60 \mathrm{mmHg})$.

Niereninsuffizienz stellt kein Ausschlusskriterium dar. Die HDCT, speziell mit Melphalan als Konditionierungsschema, wird bei Patienten mit terminaler Niereninsuffizienz i.R. der Therapie des multiplen Myeloms oder der AL-Amyloidose eingesetzt [53].
Tab. 2 Patienten der Baseler Datenbank mit PSV, die mit HDCT und ASCT behandelt wurden [30]

\begin{tabular}{|lll}
\hline Erkrankung & Patientenzahl & Response nach HDCT \\
\hline $\begin{array}{l}\text { Wegenersche } \\
\text { Granulomatose }\end{array}$ & 3 & $\begin{array}{l}\text { 3 CR, bei 2 Pat. Relapse } \\
\text { nach 2,3 bzw. 3 Jahren }\end{array}$ \\
\hline Kryoglobulinämie & 3 & $2 \mathrm{CR}$ \\
\hline M. Behçet & 3 & $1 \mathrm{CR}, 1 \mathrm{PR}$ \\
\hline Polyarteritis nodosa & 1 & \\
\hline
\end{tabular}

Innerhalb der EBMT/EULAR-Datenbank wurden 9 Patienten mit systemischer Vaskulitis erfasst (Tab. 2) [30]. Bei drei Patienten mit WG konnte zunächst eine komplette Remission erzielt werden. Davon rezidivierte die Erkrankung bei zwei Patienten nach 2,3 bzw. 3 Jahren. Bei zwei von drei Patienten mit Kryoglobulinassoziierter Vaskulitis mit sehr unterschiedlichen Krankheitsmanifestationen konnte ebenfalls eine komplette Remission erzielt werden [54]. Es ist noch unklar, ob die verschiedenen Entitäten der PSV prinzipiell unterschiedlicher Behandlungsansätze bedürfen. Nach unserer Ansicht ist es jedoch möglich, dass sowohl die Wegenersche Granulomatose als auch die mikroskopische Polyangiitis, das Churg-Strauss-Syndrom, die Panarteriitis nodosa und weitere Vaskulitiden in ähnlicher Weise auf dosiseskalierte Therapie ansprechen. Nicht die Subklassifizierung der Vaskulitis, sondern das Ausmaß und die Schwere der Erkrankung sollten die Basis der Entscheidung zu einer Hochdosis-Chemotherapie und Stammzelltransplantation bilden.

\section{Heidelberger Erfahrungen}

Im Rahmen eines an unserer Klinik entwickelten Hochdosis-Studienprotokolls für Autoimmunerkrankungen (Abb.1) wurden bisher 8 Patienten eingeschlossen, davon fünf Patienten mit PSV [55]. Hiervon wurden zwei Patienten mit Morbus Behçet und pulmonaler Beteiligung [56], ein Patient mit unklassifizierbarer Vaskulitis sowie ein Patient mit Panarteriitis nodosa (PAN) transplantiert. Drei dieser Patienten waren refraktär auf konventionelle immunsuppressive Therapie einschließlich Cyclophosphamid oder benötigten dauerhaft hohe Dosen Kortikosteroide trotz Behandlung mit Cyclophosphamid. Mit einer Beobachtungsdauer von 48 Monaten (Stand März 2003) ist ein Patient mit Morbus Behçet weiterhin in kompletter Remission, d.h. ohne jegliche klinische und radiologische Erkrankungszeichen und medikamentöse Therapie sowie Normalisierung der initial erhöhten Labor-Aktivitätsparameter (z.B. BKS, CRP). Der andere Patient mit Morbus Behçet, der wie o.g. Patient Lungenblutungen als Folge einer pulmonalen Vaskulitis aufwies (die Hälfte der Patienten mit dieser Komplikation stirbt innerhalb von drei Jahren nach Beginn der Hämoptysen [57]), erreichte eine „partielle Remission“, d.h. Besserung aller klinischen und bildgebenden Krankheitszeichen (Verschwinden der Lungenblutungen, Verschwinden der vaskulitischen Lungeninfiltrate und vaskulitischer Aktivität des ZNS im MRT), bei einer Beobachtungsdauer von 39 Monaten nach Stammzelltransplantation. Er benötigt nur noch niedrig dosierte Kortikosteroide, um milde Aktivitäts- 


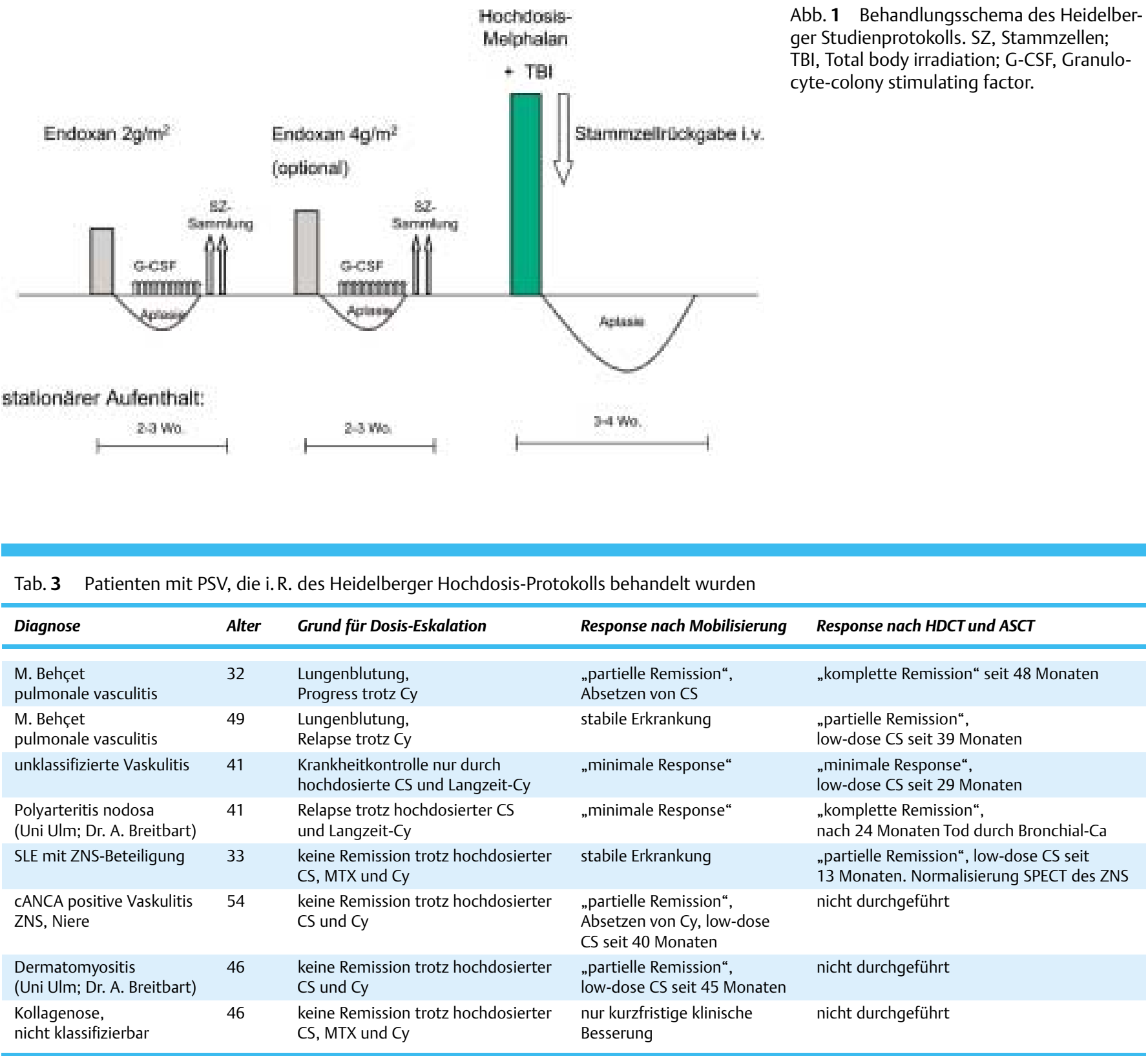

ASCT, Autologe Stammzelltransplantation; CS, Kortikosteroide; Cy, Cyclophosphamid; HDCT, Hochdosischemotherapie; MTX, Methotrexat

zeichen der Erkrankung (orale Aphthen) zu kontrollieren. Die Daten der übrigen Patienten sind in Tab. 3 zusammengefasst.

\section{Diskussion}

In wenigen Jahren ist aus einer hypothetischen und experimentellen Behandlungsform eine sehr vielversprechende, bei Hunderten von Patienten angewandte Therapieoption geworden. Erstmals steht vor allem bei der SSc eine Behandlungsmethode zur Verfügung, die den Verlauf der Erkrankung und die Behinderungen, die daraus erwachsen, günstig beeinflussen kann. Die Risiken dieser Methode müssen sorgfältig gegenüber dem zu erwartenden Nutzen abgewogen werden. Da die ASCT mit einer nicht zu vernachlässigenden Rate therapieassoziierter Nebenwirkungen bis hin zu Todesfällen behaftet ist, spielt die Patientenselektion die entscheidende Rolle bei der weiteren Entwick- lung dieser Methode in der Rheumatologie. Es müssen die Patienten ausgewählt werden, bei denen die Schwere der Erkrankung, bzw. die krankheitsassoziierte Mortalität die Toxizität der HDCT erheblich überwiegt. Leider kann auch die konventionelle immunsuppressive Therapie vor allem beim SLE und bei den PSV chronische und $z$. T. schwere Begleittoxizität mit sich bringen. Aus den Erfahrungen des National Institutes of Health bei Patienten mit der WG geht hervor, dass die Toxizität der langfristigen und hochdosierten Behandlung mit Cyclophosphamid und Kortikosteroiden (Fauci-Schema) bei über $50 \%$ der untersuchten Patienten zu einem bleibenden Schaden führt [58]. Die langfristigen Nebenwirkungen einer konventionellen „Erhaltungstherapie“ lassen sich hauptsächlich auf hohe kumulative Dosen von Cyclophosphamid zurückführen. In einer schwedischen Studie bei Patienten, die länger als ein Jahr orales Cyclophosphamid erhalten hatten, fand sich ein 11 fach erhöhtes Risiko für die Entwicklung eines Blasenkarzinoms sowie ein erhöhtes Risiko für 
Hauttumoren bei längerfristiger Azathioprin- und Steroidexposition [59]. Von 155 Patienten mit WG, die überwiegend langfristig mit Cyclophosphamid behandelt wurden, entwickelten $11 \mathrm{~Pa}-$ tienten (7\%) ein myelodysplastisches Syndrom nach einer medianen Cyclophosphamid-Dosis von $112 \mathrm{~g}, 1$ Patient entwickelte ein Blasenkarzinom [47]. Diese Komplikationen der konventionellen immunsuppressiven Therapie sind ebenfalls zu berücksichtigen und abzuwägen mit den Risiken einer ASCT. Die Toxizität des in Heidelberg favorisierten Konditionierungsregimes mit Melphalan wurde im Rahmen von Hochdosis-Programmen bei Patienten mit multiplem Myelom evaluiert [20]. Es konnte gezeigt werden, dass die Rate an Sekundärneoplasien (z. B. myelodysplastisches Syndrom, akute Leukämie) geringer war als bei lang andauernder Standard-Chemotherapie [21].

Grundsätzlich sollten für die ASCT Patienten ausgewählt werden, deren Erkrankung therapierefraktär, aktiv und progredient ist, und bei denen ein hohes Risiko für bleibende Funktionseinschränkungen und hohe Mortalität trotz konventioneller Therapie besteht. Durch verbesserte Scoring-Systeme, die eine frühere Identifikation dieser Hochrisiko-Patienten schon in frühen Krankheitsstadien erlauben, kann zukünftig der Erfolg der Methode verbessert und die Risiken minimiert werden.

Noch beruht die Erfahrung mit HDCT bei Autoimmunerkrankungen auf kleinen Fallzusammenstellungen, Phase-II-Studien und Registry-Analysen. Die beschriebenen Fälle mit überwiegend guten Ergebnissen sind vielversprechend, wenngleich nicht vergessen werden sollte, dass es sich hierbei um eine aggressive Therapieform handelt, die auch tödliche Risiken in sich birgt. Die Erfahrung mit HDCT wächst kontinuierlich. Zukünftig können allein durch multizentrische Phase-III-Studien der Nutzen und die Risiken der Stammzelltransplantation im Vergleich zur konventionellen Therapie geklärt werden. Eine erste solche kontrollierte, randomisierte Studie bei Patienten mit schwerer systemischer Sklerose wurde inzwischen gestartet (ASTIS-Trial, Autologous Stem Cell Transplantation International Scleroderma Trial; www.astistrial.com). Weitere Phase-III-Studien werden in Kürze von der EBMT und EULAR in vielen europäischen Ländern begonnen bei Patienten mit multipler Sklerose (ASTIMS Trial - Autologous Stem Cell Transplantation International Multiple Sclerosis Trial; www.ebmt.org) und schwerer, therapierefraktärer rheumatoider Arthritis (ASTIRA Trial-Autologous Stem Cell Transplantation International Rheumatoid Arthritis Trial; www. ebmt.org). Für randomisierte Studien bei primär systemischen Vaskulitiden ist es sicherlich noch zu früh. Die Erfahrung mit größeren Fallzahlen sowie längeres Follow-up bei den schon transplantierten Patienten werden jedoch helfen, die Hypothesen für zukünftige kontrollierte Studien zu entwickeln.

\section{Literatur}

${ }^{1}$ Silman AJ, Newman J. Epidemiology of systemic sclerosis. Curr Opin Rheumatol 1996; 8: 585-589

2 Bryan C, Knight C, Black CM, Silman AJ. Prediction of five-year survival following presentation with scleroderma: development of a simple model using three disease factors at first visit. Arthritis Rheum 1999; 42: $2660-2665$

${ }^{3}$ White B, Moore WC, Wigley FM, Xiao HQ Wise RA. Cyclophosphamide is associated with pulmonary function and survival benefit in patients with scleroderma and alveolitis. Ann Intern Med 2000; 132: 947-954
${ }^{4}$ Gladman DD. Indicators of disease activity, prognosis, and treatment of systemic lupus erythematosus. Curr Opin Rheumatol 1994; 6: $487-492$

${ }^{5}$ Urowitz MB, Gladman DD, Abu-Shakra M, Farewell VT. Mortality studies in systemic lupus erythematosus. Results from a single center. III. Improved survival over 24 years. J Rheumatol 1997; 24: 1061 - 1065

${ }^{6}$ Ruiz-Irastorza G, Khamashta MA, Castellino G, Hughes GR. Systemic lupus erythematosus. Lancet 2001; 357: $1027-1032$

${ }^{7}$ Noseworthy JH, Lucchinetti C, Rodriguez M, Weinshenker BG. Multiple sclerosis. N Engl J Med 2000; 343: 938 - 952

${ }^{8}$ Comi G, Moiola L. Glatiramer acetate. Neurologia 2002; 17: 244-258

${ }^{9}$ Wolinsky JS, Comi G, Filippi M, Ladkani D, Kadosh S, Shifroni G. Copaxone's effect on MRI-monitored disease in relapsing MS is reproducible and sustained. Neurology 2002; 59: 1284-1286

${ }^{10}$ Edan G, Miller D, Clanet M et al. Therapeutic effect of mitoxantrone combined with methylprednisolone in multiple sclerosis: a randomised multicentre study of active disease using MRI and clinical criteria. J Neurol Neurosurg Psychiatry 1997; 62: 112-118

${ }^{11}$ Hartung HP, Gonsette R, Konig N et al. Mitoxantrone in progressive multiple sclerosis: a placebo-controlled, double-blind, randomised, multicentre trial. Lancet 2002; 360: 2018 - 2025

12 Marmont AM. Stem cell transplantation for severe autoimmune disorders, with special reference to rheumatic diseases. J Rheumatol (Suppl) 1997; 48: $13-18$

${ }^{13}$ Ikehara S, Yasumizu R, Inaba M et al. Long-term observations of autoimmune-prone mice treated for autoimmune disease by allogeneic bone marrow transplantation. Proc Natl Acad Sci USA 1989; 86: $3306-3310$

${ }^{14}$ Knaan-Shanzer S, Houben P, Kinwel-Bohre EP, van Bekkum DW. Remission induction of adjuvant arthritis in rats by total body irradiation and autologous bone marrow transplantation. Bone Marrow Transplant 1991; 8: $333-338$

15 Slavin S. Treatment of life-threatening autoimmune diseases with myeloablative doses of immunosuppressive agents: experimental background and rationale for ABMT. Bone Marrow Transplant 1993; 12: $85-88$

${ }^{16}$ van Bekkum DW. Stem cell transplantation in experimental models of autoimmune disease. J Clin Immunol 2000; 20: 10-16

${ }^{17}$ Ikehara S. Treatment of autoimmune diseases by hematopoietic stem cell transplantation. Exp Hematol 2001; 29: 661 -669

${ }^{18}$ Schnabel A, Reuter M, Gross WL. Intravenous pulse cyclophosphamide in the treatment of interstitial lung disease due to collagen vascular diseases. Arthritis Rheum 1998; 41: 1215-1220

19 Burt RK, Fassas A, Snowden J et al. Collection of hematopoietic stem cells from patients with autoimmune diseases. Bone Marrow Transplant 2001; 28: $1-12$

${ }^{20}$ Goldschmidt H, Egerer G, Ho AD. Autologous and allogeneic stem cell transplantation in multiple myeloma. Bone Marrow Transplant 2000; 25 (Suppl 2): S25-S26

${ }^{21}$ Govindarajan R, Jagannath S, Flick JT et al. Preceding standard therapy is the likely cause of MDS after autotransplants for multiple myeloma. Br J Haematol 1996; 95: 349-353

22 Tyndall A, Fassas A, Passweg J et al. Autologous haematopoietic stem cell transplants for autoimmune disease-feasibility and transplantrelated mortality. Autoimmune Disease and Lymphoma Working Parties of the European Group for Blood and Marrow Transplantation, the European League Against Rheumatism and the International Stem Cell Project for Autoimmune Disease. Bone Marrow Transplant 1999; 24: 729-734

${ }^{23}$ Bacon PA, Carruthers D. New therapeutic aspects: haemopoietic stem cell transplantation. Best Pract Res Clin Rheumatol 2001; 15: 299 - 313

${ }^{24}$ Cobbold SP, Qin SX, Waldmann H. Reprogramming the immune system for tolerance with monoclonal antibodies. Semin Immunol 1990; 2: $377-387$

${ }^{25}$ Koehne G, Zeller W, Stockschlaeder M, Zander AR. Phenotype of lymphocyte subsets after autologous peripheral blood stem cell transplantation. Bone Marrow Transplant 1997; 19: 149-156

${ }^{26}$ Guillaume T, Rubinstein DB, Symann M. Immune reconstitution and immunotherapy after autologous hematopoietic stem cell transplantation. Blood 1998; 92: $1471-1490$

${ }^{27}$ Kozak T. Hematopoietic stem cell transplantation in the treatment of autoimmune diseases. Wien Klin Wochenschr 2002; 114: 7-13

${ }^{28}$ McKendry RJ, Huebsch L, Leclair B. Progression of rheumatoid arthritis following bone marrow transplantation. A case report with a 13-year follow-up. Arthritis Rheum 1996; 39: 1246-1253 
${ }^{29}$ Snowden JA, Kearney P, Kearney A et al. Long-term outcome of autoimmune disease following allogeneic bone marrow transplantation. Arthritis Rheum 1998; 41: 453-459

${ }^{30}$ Tyndall A, Passweg J, Gratwohl A. Haemopoietic stem cell transplantation in the treatment of severe autoimmune diseases 2000. Ann Rheum Dis 2001; 60: $702-707$

${ }^{31}$ Abu-Shakra M, Lee P. Mortality in systemic sclerosis: a comparison with the general population. J Rheumatol 1995; 22: 2100-2102

32 Altman RD, Medsger TA, Jr., Bloch DA, Michel BA. Predictors of survival in systemic sclerosis (scleroderma). Arthritis Rheum 1991; 34: $403-413$

${ }^{33}$ Hesselstrand R, Scheja A, Akesson A. Mortality and causes of death in a Swedish series of systemic sclerosis patients. Ann Rheum Dis 1998; 57: $682-686$

${ }^{34}$ Medsger TA, Stehen VD. Classification, prognosis. In: Clements PJ, Furst DE, (eds). Systemic sclerosis. Maryland: Williams \& Wilkins, 1996: $57-64$

${ }^{35}$ Tyndall A, Black C, Finke J et al. Treatment of systemic sclerosis with autologous haemopoietic stem cell transplantation. Lancet 1997; 349: 254

${ }^{36}$ Binks M, Passweg JR, Furst D et al. Phase I/II trial of autologous stem cell transplantation in systemic sclerosis: procedure related mortality and impact on skin disease. Ann Rheum Dis 2001; 60: 577-584

${ }^{37}$ McSweeney PA, Nash RA, Sullivan KM et al. High-dose immunosuppressive therapy for severe systemic sclerosis: initial outcomes. Blood 2002; 100: $1602-1610$

${ }^{38}$ Comi G, Kappos L, Clanet M et al. Guidelines for autologous blood and marrow stem cell transplantation in multiple sclerosis: a consensus report written on behalf of the European Group for Blood and Marrow Transplantation and the European Charcot Foundation. BMT-MS Study Group. J Neurol 2000; 247: 376-382

${ }^{39}$ Tyndall A. Immunoablation and haemopoietic stem cell transplantation for severe autoimmune disease with special reference to systemic lupus erythematosus. Lupus 2001; 10: $214-215$

${ }^{40}$ Musso M, Porretto F, Crescimanno A et al. Autologous peripheral blood stem and progenitor (CD34+) cell transplantation for systemic lupus erythematosus complicated by Evans syndrome. Lupus 1998; 7: $492-494$

${ }^{41}$ Fouillard L, Gorin NC, Laporte JP, Leon A, Brantus JF, Miossec P. Control of severe systemic lupus erythematosus after high-dose immunosuppressive therapy and transplantation of CD34+ purified autologous stem cells from peripheral blood. Lupus 1999; 8: 320-323

42 Wulffraat NM, Sanders EA, Kamphuis SS et al. Prolonged remission without treatment after autologous stem cell transplantation for refractory childhood systemic lupus erythematosus. Arthritis Rheum 2001; 44: 728-731

${ }^{43}$ Traynor AE, Barr WG, Rosa RM et al. Hematopoietic stem cell transplantation for severe and refractory lupus. Analysis after five years and fifteen patients. Arthritis Rheum 2002; 46: 2917-2923
${ }^{44}$ Snowden J, Moore J, Jakob R, Passweg J. Autologous stem cell transplantation in rheumatoid arthritis. Blood 2001; 98: 860a

45 Matteson EL, Gold KN, Bloch DA, Hunder GG. Long-term survival of patients with Wegener's granulomatosis from the American College of Rheumatology Wegener's Granulomatosis Classification Criteria Cohort. Am J Med 1996; 101: 129-134

${ }^{46}$ Savage CO, Winearls CG, Evans DJ, Rees AJ, Lockwood CM. Microscopic polyarteritis: presentation, pathology and prognosis. Q J Med 1985; 56: $467-483$

${ }^{47}$ Reinhold-Keller E, Beuge N, Latza U et al. An interdisciplinary approach to the care of patients with Wegener's granulomatosis: long-term outcome in 155 patients. Arthritis Rheum 2000; 43: 1021 - 1032

48 Jayne D. Update on the European Vasculitis Study Group trials. Curr Opin Rheumatol 2001; 13: 48-55

${ }^{49}$ Guillevin L, Lhote F, Gayraud M et al. Prognostic factors in polyarteritis nodosa and Churg-Strauss syndrome. A prospective study in $342 \mathrm{pa}-$ tients. Medicine (Baltimore) 1996; 75: 17-28

${ }^{50}$ Luqmani RA, Bacon PA, Moots RJ et al. Birmingham Vasculitis Activity Score (BVAS) in systemic necrotizing vasculitis. QJM 1994; 87: $671-678$

51 de Groot K, Gross WL, Herlyn K, Reinhold-Keller E. Development and validation of a disease extent index for Wegener's granulomatosis. Clin Nephrol 2001; 55: 31 - 38

52 Exley AR, Bacon PA, Luqmani RA et al. Development and initial validation of the Vasculitis Damage Index for the standardized clinical assessment of damage in the systemic vasculitides. Arthritis Rheum 1997; 40: $371-380$

${ }^{53}$ Badros A, Barlogie B, Siegel E et al. Results of autologous stem cell transplant in multiple myeloma patients with renal failure. $\mathrm{Br} J$ Haematol 2001; 114: 822-829

54 Yoon KH, Fong KY, Koh DR, Suri R. Central pontine myelinolysis - a rare manifestation of CNS Sjogren's syndrome. Lupus 2000; 9: 471 - 473

55 Jondeau K, Job-Deslandre C, Bouscary D, Khanlou N, Menkes CJ, Dreyfus F. Remission of nonerosive polyarthritis associated with Sjogren's syndrome after autologous hematopoietic stem cell transplantation for lymphoma. J Rheumatol 1997; 24: 2466 - 2468

${ }^{56}$ Hensel M, Breitbart A, Ho AD. Autologous hematopoietic stem-cell transplantation for Behçet's disease with pulmonary involvement. $\mathrm{N}$ Engl J Med 2001; $344: 69$

${ }^{57}$ Hamuryudan V, Yurdakul S, Moral F et al. Pulmonary arterial aneurysms in Behçet's syndrome: a report of 24 cases. Br J Rheumatol 1994; 33: $48-51$

${ }^{58}$ Hoffman GS, Kerr GS, Leavitt RY et al. Wegener granulomatosis: an analysis of 158 patients. Ann Intern Med 1992; 116: 488 - 498

${ }^{59}$ Westman KW, Bygren PG, Olsson H, Ranstam J, Wieslander J. Relapse rate, renal survival, and cancer morbidity in patients with Wegener's granulomatosis or microscopic polyangiitis with renal involvement. J Am Soc Nephrol 1998; 9: 842-852 